10. Соловъева Н.Ф. Современные рулонные прессподборщики // Техника и оборудование для села. 2001. - № 12. - С. 18-22.

11. Электронный ресурс: https://www.garant.ru/ products/ipo/prime/doc/71350102/ (дата обращения 06.01.2019).

12. Электронный ресурс: URL: http://www. schaumann.at/cps/schaumann-at/ds_doc/de-AT/ Dosiergeraete_Beschreibung.pdf (дата- обращения 01.02.2016).

13. Электронный ресурс: https://domaferma. com/oborudovanie/tehnologia-zagotovki-senaza-vrulonah.html (дата обращения 06.01.2019).

Кучин Николай Николаевич, $\partial-p$ c.- $\boldsymbol{x}$. наук, проф., научный сотрудник, ГБОУ ВО «Нижегородский государственный инженерно-экономический университет». Россия.
Мансуров Александр Петрович, $\partial-p$ c.- $x$. наук, проф., научный сотрудник, ИПТД филиал ГБОУ ВО «Нижегородский государственный инженерно-экономический университет». Россия.

Жужин Максим Сергеевич, канд. техн. наук, доцент кафедры «Электрификация и автоматизация», ГБОУ ВО «Нижегородский государственный инженерно-экономический университет». Россия.

Ломаченко Оксана Александровна, аспирант, ГБОУ ВО «Нижегородский государственный инженерно-экономический университет». Россия.

606360, Нижегородская область, г. Княгинино, ул. Октябрьская, 22 A.

Тел.: (83166) 4-15-50.

ключевые слова: сенаж; пленочная упаковка; заготовка кормов; сенаж в упаковке; технологчческий процесс заготовки сенажа.

\title{
PROBLEMS OF PRESERVATION OF THE SILAGE IN ROLLS, PACKED IN FILM
}

Kuchin Nikolay Nikolaevich, Doctor of Agricultural Sciences, Professor, Researcher, Nizhny Novgorod State University of Engineering and Economics. Russia.

Mansurov Alexander Petroich, Doctor of Agricultural Sciences, Professor, Researcher, Nizhny Novgorod State University of Engineering and Economics. Russia.

Zhuzhin Maxim Sergeevich, Candidate of Technical Sciences, Associate Professor of the chair "Electrification and Automation”, Nizhny Novgorod State University of Engineering and Economics. Russia.

Lomachenko Oksana Aleksandrovna, Post-graduate Student, Nizhny Novgorod State University of Engineering and

\section{Economics. Russia.}

Keywords: haylage; film packaging; fodder preparation; haylage in packaging; technological process of haylage preparation.

The article presents an overview of the operations of the technological process of preparing the seed in a roll packed in a film, as well as technical means by which these operations are performed. In the result, the advantages and disadvantages of the technology of haylage preparation in a roll packed in a film are revealed and ways of their solution are proposed.

\section{ИСПОЛЬЗОВАНИЕ ДВУХФАЗНОЙ СИСТЕМЫ СЖИЖЕННОГО УГЛЕВОДОРОДНОГО ГАЗА В СЕЛЬСКОМ ХОЗЯЙСТВЕ}

\author{
МАЛЫШЕВА Анна Александровна, Национальный исследовательский Московский \\ государственный строительный университет
}

В работе рассматривается изменение соотношения компонентов, при котором относительное содержание более легких углеводородов будет уменьшаться, а содержание более тяжелых увеличиваться. Для улучшения условий эксплуатации систем рассмотрены вопросы, связанные с расчетом двухфазной системы сжиженного углеводородного газа.

Введение. В ходе проектирования и эксплуатации установок, а также при транспортировке, хранении сжиженных углеводородных газов необходимо учитывать свойства отдельных компонентов и их смесей[4].

При интенсивном расходе газа температура жидкости будет резко падать, в результате чего при этом нарушится испарение и, как следствие, произойдет обмерзание сосуда. В связи с этим при эксплуатации баллонных установок очень важно учитывать вопросы безопасности, которые связаны с заполнением емкостей, изменением состава смеси углеводородных газов [1].
При транспортировании, хранении или регазификации углеводородные газы находятся в виде двухфазной системы, в состав которой входят жидкая и паровая фазы.

При эксплуатации следует учитывать такие свойства, как высокая плотность; медленная диффузия; низкая температура воспламенения; непостоянная теплота сгорания.

Основные свойства жидкой фазы включают в себя способность к сильному объемному сжатию и расширению, малую относительная плотность, охлаждающую способность при регазификации. 
Углеводороды, которые находятся в газообразном состоянии, способные при незначительном повышении давления переходить в жидкое состояние, относят к сжиженным углеводородным газам. В паровую фазу углеводородная жидкость может перейти при условии снижения давления, за счет чего эта жидкость испарится. Такой процесс позволяет осуществлять перевозку и хранение сжиженных углеводородов [2].

Методика исследований. Сжиженные углеводородные газы используют для нужд бытового отопления, применяют для технологических процессов в сельском хозяйстве и промышленности.

Сжиженный газ, заключенный в герметический сосуд, оказывает давление на стенки сосуда, называемое упругостью паров, которое является функцией температуры. Упругость достигается за счет большого количества легких углеводородов: чем их больше содержится в газе, тем упругость паров становится выше.

При проектировании и эксплуатации систем снабжения потребителей сжиженным газом необходимо знать некоторые термодинамические процессы, которые происходят при отборе паровой фазы [3].

Результаты исследований. В баллон поступает жидкая смесь углеводородного газа под давлением. Мольный состав смеси $\sum A=1$ (рис. 1$)$.

При этом $\mathrm{V}+\mathrm{L}=1$.

Согласно закону Дальтона, давление смеси газа будет равноценно сумме парциальных давлений компонентов:

$$
P_{\mathrm{cm}}=\sum P_{i},
$$

где $P_{i}$ - парциальное давление газа $i$, то есть такое давление, которое оказывал бы этот газ, если бы он один занимал весь объем, занимаемый смесью.

Парциальным объемом $V_{i} i$-го компонента называют объем, занимаемый им при температуре и давлении смеси.

Состав газовых смесей задают молярными долями.

Согласно закону Рауля, парциальное давление паров данного компонента, находящегося в жидкой смеси, равно его молярной концентра-

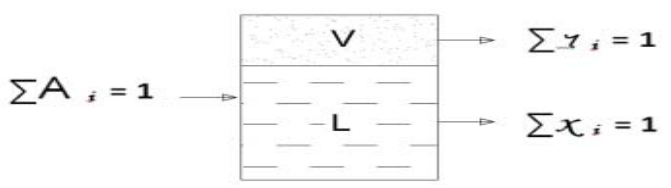

Рис. 1. Процесс разделения углеводородной смеси на жидкость и пар: V - доля от общего числа молей, находится в паровой фазе; $L$ - доля от общего числа молей, находится в жидкй фазе ции в жидкости, умноженной на упругость паров этого компонента в чистом виде при температуре смеси:

$$
P_{i}=x_{i} P_{i \text { нас, }}
$$

где $P_{i}$ - парциальное давление паров компонента $i$, находящегося в жидкой смеси; $x_{i}-$ молярная доля компонента і в жидкой смеси; $P_{i \text { нас }}-$ упругость паров чистого компонента при температуре смеси.

Из равенства парциальных давлений получаем основное уравнение

$$
\begin{gathered}
r_{i} P_{\mathrm{cm}}=x_{i} P_{i \text { нас; }} \\
A_{i}=r_{i} V+x_{i} L,
\end{gathered}
$$

где $A_{i}$ - общее число молей компонента $i$; $r_{i} V$ - число молей компонента $i$ в паровой фазе; $x_{i} L$ - число молей компонента $i$ в жидкой фазе.

Температура $t$ и давление $P$ в баллоне заданы. Требуется определить состав паровой $\sum r_{i}=1$ и жидкой фаз $\sum x_{i}=1$.

Уравнение материального баланса для компонента:

$$
A_{i}=r_{i} V+x_{i} L \text {. }
$$

Подставим значение величины $x_{i}$ :

$$
x_{i}=r_{i} \frac{P_{\mathrm{CM}}}{P_{\text {mac } i}} .
$$

В результате

$$
A_{i}=r_{i} V+\frac{r_{i} P_{\mathrm{CM}}}{P_{\text {mac } i}}(1-V) .
$$

Далее определим:

$$
r_{i}=\frac{A_{i}}{V+\frac{P_{\mathrm{CM}}}{P_{\text {Fac }} i}(1-V)} .
$$

Просуммируем $r_{i}$ для всех компонентов:

$$
\sum r_{i}=\sum \frac{A_{i}}{V+\frac{P_{\mathrm{CM}}}{P_{\text {вас } i} i}(1-V)}=1 \text {. }
$$

Из данного уравнения определим значение $V$ и рассчитаем состав паровой и жидкой фаз.

Можно получить расчетную зависимость, принимая

$$
r_{i}=x_{i} \frac{P_{i \text { Hac }}}{P_{\mathrm{CM}}} .
$$

Определим $A_{i}$ :

$$
A_{i}=x_{i} \frac{P_{i \text { Haс }}}{P_{\mathrm{CM}}}(1-L)+x_{i} L .
$$

Просуммируем $x_{i}$ для всех компонентов:

$$
\sum \mathrm{x}_{i}=\sum \frac{A_{i}}{L+\frac{P_{\mathrm{CM}}}{P_{\text {Fac }} i}(1-L)} .
$$


Из заданного уравнения определим значение $L$ и рассчитаем состав жидкой и паровой фаз.

Результаты исследования

Определим состав паровой и жидкой фаз, если начальный состав смеси:

$$
\begin{gathered}
\mathrm{A}_{\mathrm{C}_{\mathrm{g}} \mathrm{H}_{8}}=0,5 ; \\
\mathrm{A}_{\mathrm{M}_{0} 0 \mathrm{C}_{4} \mathrm{H}_{10}}=0,3 ; \\
\mathrm{A}_{\mathrm{HC}_{4} \mathrm{H}_{10}}=0,2 .
\end{gathered}
$$

Определим упругость паров компонентов смеси при $t=20^{\circ} \mathrm{C}$ в МПа (абс):

$$
\begin{aligned}
& P_{\mathrm{C}_{8} \mathrm{H}_{8}}=0,83 ; \\
& P_{{\text {и } 30 C_{4} \mathrm{H}_{10}}}=0,306 ; \\
& P_{\mathrm{Hc}_{4} \mathrm{H}_{10}}=0,23 .
\end{aligned}
$$

Рассчитаем давление смеси:

$0,5 \cdot 0,83+0,3 \cdot 0,306+0,2 \cdot 0,23=0,553$ Мпа.

Найдем значение $\sum x_{i}=1$ :

$$
\begin{gathered}
\sum x_{i}=\sum \frac{A_{i}}{V+\frac{P_{\mathrm{CM}}}{P_{\operatorname{mac} i} i}(1-V)}=1 ; \\
\frac{0,5}{V+\frac{0,558}{0,88}(1-V)}+\frac{0,3}{V+\frac{0,558}{0,806}(1-V)}+\frac{0,2}{V+\frac{0,558}{0,28}(1-V)}=1 .
\end{gathered}
$$

Решив это выражение, получим $V=0,49$.

Определим состав паровой смеси:

$$
\begin{gathered}
r_{\mathrm{c}_{\mathrm{g}} \mathrm{H}_{\mathrm{g}}}=\frac{0,5}{0,49+\frac{0,558}{0,88} 0,51}=\frac{0,5}{0,49+0,667 \cdot 0,51}=0,65 ; \\
r_{\mathrm{U}_{00 \mathrm{C}_{4} \mathrm{H}_{10}}}=\frac{0,3}{0,49+\frac{0,558}{0,806} 0,51}=\frac{0,3}{0,49+1,807 \cdot 0,51}=0,22 ; \\
r_{\mathrm{Hc}_{4} \mathrm{H}_{10}}=\frac{0,2}{0,49+\frac{0,558}{0,28} 0,51}=\frac{0,2}{0,49+2,4 \cdot 0,51}=0,13 .
\end{gathered}
$$

Определим состав жидкой фазы:

$$
\begin{gathered}
x_{i}=\frac{r_{i} P_{\mathrm{CM}}}{P_{i \mathrm{Hac}}} ; \\
x_{\mathrm{Cg}_{\mathrm{g}} \mathrm{H}}=\frac{0,65 \cdot 0,553}{0,83}=0,4 ; \\
x_{{\mathrm{и} 30 \mathrm{C}_{4} \mathrm{H}_{10}}}=\frac{0,22 \cdot 0,553}{0,306}=0,3 ; \\
x_{\mathrm{Hc}_{4} \mathrm{H}_{10}}=\frac{0,13 \cdot 0,553}{0,23}=0,3 .
\end{gathered}
$$

Заключение. На основании вышеизложенного можно заключить, что при отводе паровой фазы из баллона нужно учитывать наружные равновесия, которые происходят между жидкостью и газом. Рассмотрены вопросы изменения соотношения компонентов, при котором относительное содержание более легких углеводородов будет уменьшаться, а содержание более тяжелых увеличиваться.

При интенсивном расходе газа температура жидкости будет резко падать, в результате чего при этом нарушится испарение и, как следствие, произойдет обмерзание сосуда. Это явление особенно часто наблюдается при эксплуатации газобаллонных установок, то есть с увеличением расхода сжиженного газа из них выходит пар. Испарительная способность также зависит от степени заполнения баллона. Чем больше смачиваемая поверхность, тем больше испарительная способность. В связи с этим при эксплуатации баллонных установок очень важно учитывать вопросы безопасности, которые связаны с заполнением емкостей, изменением состава смеси углеводородных газов.

\section{Список литературы}

1. ГОСТ Р 54961-2012. Системы газораспределительные. Сети газопотребления. Общие требования к эксплуатации. Эксплуатационная документация. - М., 2012.

2. Жила В.А., Соловьева Е.Б., Гулюкин М.Д. Безопасность систем газоснабжения и газораспределения // Научное обозрение. - 2016. - № 22. - С. 27-32.

3. СП 62.13330.2011. Газораспределительные системы. - М., 2011. - Режим доступа: http://docs.cntd. $\mathrm{ru} /$ document/1200084535.

4. Solovyeva E. Evaluation of the safety of gas distribution systems, taking into account potential gas leakage from gas pipelines of high and medium pressure. In: International Journal of Mechanical Engineering \& Technology (IJMET). Scopus Indexed. 2018, Vol. 9, Iss. 2, P.761-764.

Малышева Анна Александровна, канд. техн. наук, доцент кафедры «Теплогазоснабжение и вентиляция», Национальный исследовательский Московский государственный строительный университет. Россия.

129337, г. Москва, Ярославское шоссе, 26.

Тел.: (495) 781-80-07.

Ключевые слова: газораспределительные системы; сельское хозяйство; углеводородный газ.

\section{THE USE OF A TWO-PHASE SYSTEM OF LIQUEFIED PETROLEUM GAS IN AGRICULTURE}

Malysheva Anna Aleksandrovna, Candidate of Technical Sciences, Associate Professor of the chair "Heat and Gas Supply and Ventilation", National Research Moscow State University of Civil Engineering. Russia.

Keywords: gas distribution systems; agriculture; carbon-hydrogen gas.
The article considers a change in the ratio of components in which the relative content of lighter hydrocarbons will decrease, and the content of heavier hydrocarbons will increase. To improve the operating conditions of the systems, issues related to the calculation of a two-phase system of liquefied carbon-hydrogen gas are considered. 